lar spectrum would hide any stellar lines of the same wave lengths.

Visual observations of the nova at the Cassegrain focus of the 100 -inch reflector with excellent seeing clearly showed the elongation of the nebula in P.A. $135^{\circ}$, but no duplicity was noted.

CARnegie Institution of Washington MOUnt Wilson ObSERvatory

October 1940

THE SPECTRUM OF P CYGNI IN THE REGION $\lambda 3000-\lambda 3300$

By P. Swings and O. Struve

The spectrum of P Cygni from $\lambda 3000$ to $\lambda 3300$ has recently been photographed with the quartz spectrograph of the McDonald Observatory. The average dispersion in this region is 15 $\mathrm{A} / \mathrm{mm}$. The lines are shown in Table $\mathrm{I}$.

The first line, $\lambda 3013$, is a strong feature; there may also be a bright line of shorter wave length, at $\lambda 3008$, due to $F e$ III, but this is not certain. The spectrum of P Cygni below $\lambda 3300$

TABLE I

Spectrum of P Cygni in the Region $\lambda<3300$

\begin{tabular}{|c|c|c|c|c|c|}
\hline & \multirow{2}{*}{$\lambda$} & \multirow{2}{*}{ Intensity } & \multicolumn{3}{|c|}{ Identification } \\
\hline & & & Element & $\lambda$ & Intensity \\
\hline 3011.93 & .. & 4A) & & & \\
\hline 3012.98 & $\ldots$ & $4 \mathrm{E}\}$ & $F e$ III & 3013.12 & 20 \\
\hline 3023.63 & $\ldots \ldots$ & $1 \mathrm{E}^{\prime}$ & Fe III & 3023.85 & 8 \\
\hline 3085.33 & $\ldots$ & $6 \mathrm{~A}$ & $S i$ III & 3086.22 & 7 \\
\hline 3092.52 & $\ldots \ldots \ldots \ldots$ & $5 \mathrm{~A}$ & $S i$ III & 3093.42 & 6 \\
\hline 3095.85 & $\ldots \ldots \ldots$ & $2 \mathrm{~A}$ & $S i$ III & 3096.79 & 4 \\
\hline 3108.90 & $\ldots$ & $1 \mathrm{~A}$ & $\mathrm{Fe}$ III & 3110.05 & 10 \\
\hline 3110.64 & $\ldots$ & $1 \mathrm{~A}$ & $\mathrm{Fe}$ III & 3111.61 & 8 \\
\hline 3117.48 & $\ldots$. & $1 \mathrm{~A}$ & $F e$ III & 3118.75 & 5 \\
\hline 3131.46 & $\ldots$ & $1 \mathrm{~A}$ & $? 0 \mathrm{III}$ & 3132.86 & 6 \\
\hline 3134.96 & .. & $3 \mathrm{~A}$ ) & & & \\
\hline 3136.28 & . & $3 \mathrm{E} \int^{\zeta}$ & $F e$ III & 3136.43 & 10 \\
\hline 3165.46 & .. & $1 \mathrm{~A}^{J}$ & $S i \mathrm{IV}$ & 3165.72 & 8 \\
\hline 3168.27 & $\ldots$ & $1 \mathrm{~A}$ & $F e$ III & 3169.4 & 3 \\
\hline 3172.91 & $\ldots$ & $2 \mathrm{~A})$ & & & \\
\hline 3173.67 & .. & $2 \mathrm{ES}$ & $F e$ III & 3174.09 & 10 \\
\hline 3174.94 & . & $2 \mathrm{~A}\}$ & & & \\
\hline 3175.66 & $\ldots$ & $2 \mathrm{E}\}$ & Fe III & 3176.00 & 10 \\
\hline
\end{tabular}


TABLE I (continued)

\begin{tabular}{|c|c|c|c|c|c|}
\hline & \multirow{2}{*}{$\lambda$} & \multirow{2}{*}{ Intensity } & \multicolumn{3}{|c|}{ Identification } \\
\hline & & & Element & $\lambda$ & Intensity \\
\hline 3176.96 & $\cdots$ & $2 \mathrm{~A}\}$ & & & \\
\hline 3177.85 & $\ldots$ & $2 \mathrm{E}\}$ & $F e$ III & 3178.03 & 10 \\
\hline 3183.41 & $\ldots$ & $0 \mathrm{~A}\{$ & & & \\
\hline 3184.76 & $\ldots \ldots \ldots \ldots$ & $1 \mathrm{Ej}$ & Si III & 3185.16 & 3 \\
\hline 3185.94 & $\ldots \ldots$ & $10 \mathrm{~A}\}$ & & & \\
\hline 3187.42 & $\ldots$ & $10 \mathrm{E}$ & HeI & 3187.74 & 8 \\
\hline 3203.30 & $\ldots \ldots$ & $1 \mathrm{~A}\}$ & & & \\
\hline 3204.47 & . & $1 \mathrm{E}$ & Fe III & 3204.76 & 6 \\
\hline 3210.48 & . & $1 \mathrm{~A}\}$ & & & \\
\hline 3212.00 & .. & $1 \mathrm{E}\}$ & $\mathrm{Fe}$ III & 3212.05 & 10 \\
\hline 3214.07 & $\ldots$ & $1 \mathrm{~A}\}$ & & & \\
\hline 3215.31 & $\ldots$ & $1 \mathrm{E}\}$ & $\mathrm{Fe}$ III & 3215.60 & 8 \\
\hline 3260.98 & $\ldots$ & $0 \mathrm{~A}$ & $\mathrm{Fe}$ III & 3262.44 & 6 \\
\hline 3265.12 & $\cdots$ & $10 \mathrm{~A}\}$ & & & \\
\hline 3266.60 & .. & $10 \mathrm{E}\}$ & $F e$ III & 3260.88 & 20 \\
\hline 3274.52 & . & $8 \mathrm{~A}\}$ & $F_{e}$ IUt & 327608 & 15 \\
\hline 3276.13 & .. & $8 \mathrm{E} 5$ & re in & $52 / 0.00$ & 15 \\
\hline 3280.78 & .. & $O \mathrm{E}^{\prime}$ & $F e$ III & 3280.58 & 6 \\
\hline 3287.21 & .. & $6 \mathrm{~A})$ & Fe UT & 328881 & 15 \\
\hline 3288.51 & . & $6 \mathrm{E}\{$ & re 11 & 3280.81 & 10 \\
\hline 3290.62 & $\ldots \ldots \ldots$ & $2 \mathrm{~A}\}$ & $F_{e}$ UIT & 320204 & 8 \\
\hline 3291.75 & & $2 \mathrm{E}\}$ & ге 11 & 5292.04 & 8 \\
\hline
\end{tabular}

consists of one strong line of $\mathrm{He}$ I $\left(\lambda 3188 ; 2 \mathrm{~s}^{3} \mathrm{~S}-4 \mathrm{p}^{3} \mathrm{P}^{\circ}\right)$, four lines of $S i$ III $\left(3 \mathrm{~d}^{3} \mathrm{D}-4 \mathrm{p}^{3} \mathrm{P}^{\circ}\right.$ and $\left.4 \mathrm{p}^{1} \mathrm{P}^{\circ}-5 \mathrm{~s}^{1} \mathrm{~S}\right)$ and nineteen lines of $F e$ III (mostly a ${ }^{5} \mathrm{~F}-z^{5} \mathrm{~F}^{\circ} ; a^{3} \mathrm{~F}-z^{3} \mathrm{D}^{\circ}$; a ${ }^{3} \mathrm{~F}-z^{3} \mathrm{G}^{\circ}$; a $\left.{ }^{5} \mathrm{~F}-z^{5} \mathrm{G}^{0}\right)$. All the strong observed lines of $F e$ III have metastable lower levels. $\mathrm{He}$ I ( $\lambda$ 3188) also has a lower metastable level. The $S i$ III lines observed in this region confirm and complete the peculiar selectivity observed in other spectral regions: $:^{1}$ all the observed transitions of $S i$ III, for which the term $4 \mathrm{p}^{1,3} \mathrm{P}^{\circ}$ is the upper level, are present in absorption; whereas those transitions for which this term $4 \mathrm{p}^{1,3} \mathrm{P}^{\circ}$ is the lower level are in emission. This selectivity has been discussed previously. ${ }^{1}$

\section{McDonald Observatory}

September 1940

${ }^{1}$ O. Struve and F. E. Roach, Ap. J., 90, 727, 1939; P. Swings and O. Struve; ibid., 91, 574, 1940. 